\title{
PERIODIC SOLUTIONS OF A SYSTEM OF NONLINEAR DIFFERENTIAL EQUATIONS
}

\section{ST ANISKAW SẸDZIWY ${ }^{1}$}

ABSTRACT. The paper consists of a study of the existence of periodic solutions of a system of differential equations using Borsuk's theorem on odd mappings. Applications are given to $n$th order nonlinear vector differential systems and $n$th order nonlinear scalar differential equations.

1. Introduction. This note concern $s$ the existence of $\omega$-periodic solutions of the system of differential equations

$$
y^{\prime}=A y+F(t, y)
$$

where $y \in R^{n}$ ( $R^{n}$ is real Euclidean $n$-space), $A$ is a constant $n \times n$ matrix and $F: R \times R^{n} \rightarrow R^{n}$ is continuous, $\omega$-periodic in $t$, and $F$ is not necessarily small.

This problem in the case of a nonsingular $A$ has been investigated by M. A. Krasnosel'skii [1], V. A. Pliss [2], and others. The present paper generalizes results of the above papers to the case of matrices with multiple characteristic root $\lambda_{0}=0$. Partial results in this direction have been obtained by the author [8], [9] and R. Reissig (see [3], [4], for example) in connection with the investigation of the behavior of solutions of $n$th order nonlinear equations.

In contrast to previous papers, the proofs presented here are based on an application of Borsuk's the orem on odd mappings to the translation operation generated by (1.1).

The main result of the paper is stated and proved in $\$ 2$. In $\$ 3$, applications of the main result are discussed.

The following notation will be used. The norm in $R^{n}$ is denoted by |1. If $B$ is an $m \times n$ matrix, $B^{T}$ denotes the matrix transpose of $B$. If

Received by the editors January 2, 1974.

AMS (MOS) subject classifications (1970). Primary 34C25.

Key words and phrases. Periodic solutions, Borsuk's theorem, system of ordinary nonlinear differential equations.

1This paper was written while the author was visiting the University of Utah, Autumn 1973. 
$A$ is square, $A^{-1}$ denotes the inverse and $|A|$ the norm, $\max \{|A x|:|x|=1\}$, of $A$. The same notation will be used for norms in spaces of lower dimensions. $I_{k}$ denotes the $k \times k$ unit matrix. If $C \subset R^{n}$, then $\bar{C}$, Int $C$, and $\mathrm{Bd} C$ will stand for the closure, the interior, and the boundary of $C$. Recall that $C \subset R^{n}$ is a cone (with vertex at the origin) if $\lambda C \equiv\{\lambda x: x \in C\} \subset C$ for $\lambda \geq 0$.

\section{Existence of periodic solutions of first order systems.}

Theorem 1. Assume $\lambda_{0}=0$ is a $k$-fold $(k \leq n)$ characteristic root of $A$, and let the remaining roots be different from $2 p \pi i / \omega, p=1,2, \ldots$. Let $A$ have $k$ linearly independent eigenvectors $\left\{m_{i}\right\}_{i=1}^{k}$ corresponding to $\lambda_{0}$. Let $F: R \times R^{n} \rightarrow R^{n}$ be continuous and $\omega$-periodic in $t$, and let

$$
|F(t, y)| \leq \mu_{0}|y| \text { for }|y| \geq y_{0} \text { and } t \in[0, \omega] \text {. }
$$

Suppose there exists an $n \times k$ matrix $N$ such that $A^{T} N=0, \operatorname{rank}(N)=$ $k$ and

$$
y^{T} N N^{T} F(t, y) \leq 0 \text { for all } y \in C,|y| \geq y_{1}, t \in[0, \omega],
$$

where $C$ is a cone satisfying $\left\{y: y=\sum_{i=1}^{k} \lambda_{i} m_{i}, \Sigma_{i=1}^{k}\left|\lambda_{i}\right|>0\right\} \subset$ Int $C$.

Then (1.1) has at least one $\omega$-periodic solution provided $\mu_{0}$ is sufficiently small.

Proof. Let $P$ be a nonsingular $n \times n$ matrix having $m_{i}(i=1, \cdots, k)$ as its last columns. The linear change of coordinates, $y=P x$, transforms (1.1) into the system

$$
x_{1}^{\prime}=D x_{1}+f_{1}(t, x), \quad x_{2}^{\prime}=f_{2}(t, x)
$$

where

$$
x=\left(\begin{array}{l}
x_{1} \\
x_{2}
\end{array}\right), \quad x_{1} \in R^{n-k}, \quad\left(\begin{array}{l}
f_{1}(t, x) \\
f_{2}(t, x)
\end{array}\right)=f(t, x)=P^{-1} F(t, P x) ;
$$

then (2.1) and (2.2) assume the form

$$
\begin{gathered}
|f(t, x)| \leq \mu|x| \text { for }|x| \geq r_{0}, t \in[0, \omega], \\
x_{2}^{T} H f_{2}(t, x) \leq 0 \text { for }|x| \geq r_{1}, t \in[0, \omega], x \in C_{1} ;
\end{gathered}
$$

where $\left(\begin{array}{ll}0 & 0 \\ 0 & H\end{array}\right)=P^{T} N N^{T} P$ and $C_{1}$ is the cone $\{x: P x \in C\}$. Since $\operatorname{rank}(N)=k$, $H$ is positive definite, i.e. $w^{T} H w>0$ for all $w \in R^{k}, w \neq 0$. For $w \in R^{k}$ let $\|w\|^{2}=w^{T} H w$, and for $y>0$ put $C(y)=\left\{x_{:}:\left|x_{1}\right|<y\left\|x_{2}\right\|\right\}$. Since Int $C_{1}$ 
contains the set $\left\{x: x_{1}=0, x_{2} \neq 0\right\}$, a compactness argument with the set $\left|x_{2}\right|=1$ shows $C(\gamma) \subset \operatorname{Int}\left(C_{1}\right)$ for all small $\gamma>0$. Fix $\gamma$.

Assume for the moment that $f_{2}$ satisfies (2.5) with the strict inequality sign, and let initial value problems for (2.3) have unique solutions. Associated with (2.3) is the mapping $T$ of $R^{n}$ into itself (the translation operator of (2.3)) defined by $T\left(x_{0}\right)=x\left(\omega, x_{0}\right)$, where $x=x\left(t, x_{0}\right)=\left(x_{1}\left(t, x_{0}\right), x_{2}\left(t, x_{0}\right)\right)$ denotes the solution of the initial value problem (2.3), with initial conditions $x_{1}(0)=x_{1}^{0}, x_{2}(0)=x_{2}^{0}\left(x_{0}=\left(x_{1}^{0}, x_{2}^{0}\right)\right)$. Clearly $T$ is continuous, and (2.3) has an $\omega$-periodic solution if and only if $T$ has a fixed point. For the existence proof we will construct an open bounded set ' $G \subset R^{n}$ such that $0 \in G, G=-1 \cdot G$ (i.e. ' $G$ is symmetric) and

$$
(T-e)(x) \neq 0 \quad \text { for } x \in \mathrm{Bd} G,
$$

$$
\beta(T-e)(x) \neq(1-\beta)(T-e)(-x) \text { for } x \in \mathrm{Bd} G, \beta \in[1 / 2,1] \text {, }
$$

where $e$ is the identity map. By Borsuk's theorem [7, Corollary 3.31, p. 82], this will imply that $T$ has a fixed point in ' $G$.

First we will prove that there exist positive numbers $\mu_{1}, \gamma_{1}, r_{2}, r_{3}$ such that, for all $\mu \in\left[0, \mu_{1}\right]$, if $\left\|x_{2}^{0}\right\| \geq r_{2}$ and $x_{0} \in C\left(\gamma_{1}\right)$ then

$$
\left|x\left(t, x_{0}\right)\right|>r_{1} \text { and } x\left(t, x_{0}\right) \in C(\gamma) \text { for } t \in[0, \omega]
$$

and, if $\left|\dot{x}_{1}^{0}\right| \geq r_{3}$ and $x_{0} \notin C\left(\gamma_{1}\right)$, then

$$
x_{1}\left(\omega, x_{0}\right) \neq x_{1}^{0} .
$$

In order to see this, note first that (2.4) implies that

$$
|f(t, x)| \leq \alpha+\mu|x| \text { for }(t, x) \in R \times R^{n},
$$

where $\alpha$ is a suitable constant.

Let $c=\max \left\{\left|e^{D} t\right|+1: t \in[0, \omega]\right\}$. By (2.3),

$$
\begin{aligned}
& \left.x_{1}\left(t, x_{0}\right)=e^{D t} x_{1}^{0}+\int_{0}^{t} e^{D(t-s)} f_{1}\left(s, x_{0}\right)\right) d s, \\
& x_{2}\left(t, x_{0}\right)=x_{2}^{0}+\int_{0}^{t} f_{2}\left(s, x\left(s, x_{0}\right)\right) d s .
\end{aligned}
$$

Since the norm of the matrix

$$
\left(\begin{array}{cc}
e^{D t} & 0 \\
0 & I_{k}
\end{array}\right)
$$

does not exceed $c,(2.10)$ and (2.11) imply that 


$$
\left|x\left(t, x_{0}\right)\right| \leq c\left|x_{0}\right|+\int_{0}^{t} c\left(\alpha+\mu\left|x\left(s, x_{0}\right)\right|\right) d s .
$$

Hence by Gronwall's inequality

$$
\left|x\left(t, x_{0}\right)\right| \leq c\left(\left|x_{0}\right|+\alpha \omega\right) e^{c \mu t} \text { for } t \in[0, \omega] .
$$

Let $c_{1}=\max \left\{\left|e^{D t}-I_{n-k}\right|: t \in[0, \omega]\right\}, y_{i}=x_{i}\left(t, x_{0}\right)(i=1,2)$, and denote by $h_{1}^{2}, h_{2}^{2}$, respectively, the largest and the smallest eigenvalue of H.

From (2,12) and inequalities

$$
\begin{aligned}
& \left|y-x_{0}\right| \leq\left|y_{1}-x_{1}^{0}\right|+\left|y_{2}-x_{2}^{0}\right|, \\
& \left|y_{1}-x_{1}^{0}\right| \leq c_{1}\left|x_{1}^{0}\right|+\int_{0}^{t} c\left(\alpha+\mu\left|x\left(s, x_{0}\right)\right|\right) d s, \\
& \left|y_{2}-x_{2}^{0}\right| \leq \int_{0}^{t}\left(\alpha+\mu\left|x\left(s, x_{0}\right)\right|\right) d s,
\end{aligned}
$$

it follows that

$$
\left|y-x_{0}\right| \leq k_{1}(\mu)\left|x_{1}^{0}\right|+\left(k_{2}(\mu)+k_{3}(\mu) r_{2}^{-1}\right)\left|x_{2}^{0}\right| \text { for } t \in[0, \omega],\left|x_{2}^{0}\right| \geq r_{2} \text {, }
$$

where $k_{2}(\mu)=(c+1)\left(e^{\mu c \omega}-1\right), k_{1}(\mu)=c_{1}+k_{2}(\mu)$ and $k_{3}(\mu)=$ $a \omega\left[1+c+k_{2}(\mu)\right]$.

If $x_{0} \in C\left(\gamma_{1}\right)$, then $\left|x_{1}^{0}\right| \leq \gamma_{1} h_{1}\left|x_{2}^{0}\right|$. The preceding inequalities together with $\left|y_{1}\right| \leq\left|y-x_{0}\right|+\left|x_{1}^{0}\right|,\left|x_{2}^{0}\right| \leq\left|y-x_{0}\right|+\left|y_{2}\right|$ imply

$$
\begin{aligned}
\left|y_{1}\right| \leq\left[\left(k_{1}(\mu)+1\right) \gamma_{1} b_{1}+k_{2}(\mu)+k_{3}(\mu) r_{2}^{-1}\right]\left|x_{2}^{0}\right| \equiv m_{1}\left|x_{2}^{0}\right|, \\
\left|x_{2}^{0}\right|\left[1-k_{1}(\mu) \gamma_{1} b_{1}-k_{2}(\mu)-k_{3}(\mu) r_{2}^{-1}\right] \equiv\left|x_{2}^{0}\right| m_{2} \leq\left|y_{2}\right| .
\end{aligned}
$$

Let $r_{2}$ be so large that $r_{2}>h_{1} r_{1}+2 k_{3}(0)$ and $k_{3}(0)\left[r_{2}-k_{3}(0)\right]^{-1}<$ $\gamma h_{2}$.

Then, by continuity, there exist $\gamma_{1}>0$ and $\mu_{0}>0$ such that for $\mu \epsilon$ $\left[0, \mu_{1}\right]$

$$
m_{1} \cdot m_{2}^{-1}<y b_{2}, \quad b_{1}^{-1}\left(m_{2}-m_{1}\right) r_{2}>r_{1},
$$

which proves (2.8).

To show (2.9), note that $x_{1}\left(\omega, x_{0}\right)-x_{1}^{0}=0$ is equivalent to

$$
\left(e^{D \omega}-I_{n-k}\right) x_{1}^{0}=-\int_{0}^{\omega} e^{D(\omega-s)} f_{1}\left(s, x\left(s, x_{0}\right)\right) d s .
$$

Since characteristic roots of $D$ are different from $2 p \pi i / \omega(p=1,2, \ldots)$, the matrix $e^{D \omega}-I_{n-k}$ is nonsingular, which implies that there is an $l>0$ such that $l|w| \leq\left|\left(e^{D} \omega-I_{n-k}\right) w\right|$ for all $w \in R^{n-k}$. 
Since $\left\|x_{2}\right\| \geq h_{2}\left|x_{2}\right|, x_{0} \notin C\left(\gamma_{1}\right)$ implies that $\gamma_{1} h_{2}\left|x_{2}^{0}\right| \leq\left|x_{1}^{0}\right|$. Hence $\left|x_{0}\right|<\left(1+1 / h_{2} \gamma_{1}\right)\left|x_{1}^{0}\right|$ and we get finally

$$
l\left|x_{1}^{0}\right| \leq c \alpha \omega e^{\mu c \omega}+c\left(1+\frac{1}{b_{2} \gamma_{1}}\right)\left|x_{1}^{0}\right|\left(e^{\mu c \omega}-1\right) .
$$

Replacing $\mu_{1}$ by a smaller number if necessary, we may assume that $c\left(1+1 / h_{2} \gamma_{1}\right)\left(e^{\mu_{1} c \omega}-1\right)<l$. So from (2.13) it follows that if $x_{1}^{0}$ satisfies $x_{1}\left(\omega, x_{0}\right)-x_{1}^{0}=0$, then $\left|x_{1}^{0}\right|$ is bounded, which proves (2.9).

Now let $G$ be the set $\left\{x:\left|x_{1}\right|<r_{3}\right\} \cap\left\{x:\left\|x_{2}\right\|<r_{2}\right\}$ where $r_{2}$ and $r_{3}$ are defined as above. We have $B d G=F_{1} \cup F_{2}$, where $F_{1}=\left\{x_{:}\left|x_{1}\right|=r_{3}\right.$, $\left.\left\|x_{2}\right\| \leq r_{2}\right\}$ and $F_{2}=\left\{x:\left|x_{1}\right|<r_{3},\left\|x_{2}\right\|=r_{2}\right\}$.

Increasing $r_{2}$ or $r_{3}$ if necessary, we may assume that $r_{3}=\gamma_{1} r_{2}$. Then $\gamma\left\|x_{2}^{0}\right\| \leq\left|x_{1}^{0}\right|$ for $x_{0} \in F_{1}$, hence $x_{0} \notin C\left(\gamma_{1}\right)$. Thus (2.9) implies (2.6) for $x_{0}^{1} \in F_{1}$.

Let $x_{0} \in F_{2}$. Put $W(t)=\left\|x_{2}\left(t, x_{0}\right)\right\|$. Since $F_{2} \subset C\left(\gamma_{1}\right)$ and

$$
W^{\prime}(t)=\frac{1}{W(t)} x_{2}^{T}\left(t, x_{0}\right) H f_{2}\left(t, x\left(t, x_{0}\right)\right),
$$

(2.5) and (2.8) imply that $W^{\prime}(t)<0$ for $t \in[0, \omega]$; hence

$$
\left\|x_{2}\left(\omega, x_{0}\right)\right\|<\left\|x_{2}^{0}\right\| \text {, }
$$

which completes the proof of (2.6).

From (2.14) it is easy to verify (2.7), for $x_{0} \in F_{2}$.

Assume $x_{0} \in F_{1}$, and (2.7) fails for some $\beta \in[1 / 2,1]$. By (2.11),

$$
\begin{aligned}
\left|\left(e^{D \omega}-I_{n-k}\right) x_{1}^{0}\right| \leq \beta & \int_{0}^{\omega} c\left(\alpha+\mu\left|x\left(s, x_{0}\right)\right|\right) d s \\
& +(1-\beta) \int_{0}^{\omega} c\left(\alpha+\mu\left|x\left(s,-x_{0}\right)\right|\right) d s,
\end{aligned}
$$

and by arguments used in the proof of (2.9), we conclude that $\left|x_{1}^{0}\right|<r_{3}$, which contradicts $x_{0} \in F_{1}$.

Thus $G$ has properties (2.6), (2.7), and reference to Borsuk's theorem proves the theorem under our special assumptions of uniqueness and strict inequality in (2.5).

The general case is obtained by uniform approximation of $f$ on $[0, \omega] \times$ $\bar{G}$ and a standard limiting argument (see [10], for example). The details are left to the reader.

3. Periodic solutions of $n$th order systems. As an application of Theorem 1, we will consider the problem of existence of periodic solutions 
of the $n$th order system of differential equations

$$
y^{(n)}+A_{1} y^{(n-1)}+\cdots+A_{n-1} y^{\prime}+f(y)=p(t),
$$

where $y$ is an $m$-vector, the $A_{i}$ are constant $m \times m$ matrices, and $f: R^{m}$ $\rightarrow R^{m}, p: R \rightarrow R^{m}$ are continuous.

Theorem 2. Let $A_{n-1}$ be symmetric and positive definite and let the roots of the polynomial $\phi(\lambda)=\operatorname{det}\left(\lambda^{n-1} I_{m}+\lambda^{n-2} A_{1}+\cdots+A_{n-1}\right)$ satisfy $\lambda_{i} \neq(2 \pi i / \omega) p(p=1,2, \cdots)$. Assume that

$$
\begin{gathered}
|f(y)| \leq \mu_{0}|y| \quad \text { for }|y| \geq y_{0}, \\
y^{T} f(y) \geq k|y||f(y)| \quad(0<k \leq 1) \text { for all } y .
\end{gathered}
$$

Let $p$ be $\omega$-periodic and satisfy one of the conditions:

$$
\int_{0}^{\omega} p(s) d s=0
$$

or

$$
\min \left\{k|f(y)|:|y| \geq y_{1}\right\}>p_{2}=\max \{|p(t)|: t \in[0, \omega]\}
$$

Then (3.1) has an $\omega$-periodic solution, provided $\mu_{0}$ is small enough.

A weaker version of this theorem was proved in [9]. Theorem 2 with (3.4) gives an extension of results of R. Reissig [3], [4] where the existence of periodic solutions was proved under the hypotheses that $\lim _{|y| \rightarrow \infty}|f(y)| /|y|$ $=0$ and that $\phi$ has roots with negative real parts.

Proof. Let (3.4) hold. Replace (3.1) by the equivalent system

$$
x^{\prime}=F x+b z+b p_{1}(t), \quad z^{\prime}=-f\left(c^{T} x\right),
$$

where $p_{1}(t)=\int_{0}^{t} p(s) d s, x^{T}=\left(x_{1} \cdots x_{n-1}\right), x_{i} \in R^{m}, x_{1}=y$ and $b, c, F$ are the $(n-1) m \times m,(n-1) m \times m,(n-1) m \times(n-1) m$ matrices

$$
b=\left(\begin{array}{l}
0 \\
\vdots \\
0 \\
I_{m}
\end{array}\right), \quad c=\left(\begin{array}{c}
I_{m} \\
0 \\
\vdots \\
0
\end{array}\right), \quad A=\left(\begin{array}{lllll}
0 & I_{m} & 0 & \cdots & 0 \\
0 & 0 & I_{m} & \cdots & 0 \\
0 & 0 & 0 & \cdots & 0 \\
\vdots & \vdots & \vdots & & \vdots \\
0 & 0 & 0 & \cdots & I_{m} \\
-A_{n-1} & -A_{n-2} & -A_{n-3} & \cdots & -A_{1}
\end{array}\right) .
$$


Then (3.6) is of the form (1.1) with

$$
A=\left(\begin{array}{ll}
F & b \\
0 & 0
\end{array}\right), \quad F(t, x, z)=\left(\begin{array}{c}
b p_{1}(t) \\
-f\left(c^{T} x\right)
\end{array}\right),
$$

so it suffices to verify the assumptions of Theorem 1. Put

$$
M=\left(\begin{array}{c}
-F^{-1} b \\
I_{m}
\end{array}\right) \text {. }
$$

Since $\operatorname{det}\left(A-\lambda I_{m n}\right)=\lambda^{m}(-1)^{m(n-1)} \phi(\lambda)$, rank $(M)=m, A M=0$, the matrix $A$ satisfies the conditions of Theorem 1 .

By (3.2) and (3.3), $F(t, x, z)$ satisfies (2.1). Let $K$ be a symmetric matrix such that $K^{2}=A_{n-1}^{-1}$. Define the $n m \times m$ matrix $N$ by $N=\left(\begin{array}{l}0 \\ K\end{array}\right)$. With the above notation, (2.2) may be written as

$$
-z^{T} K^{2} f\left(c^{T} x\right) \leq 0 .
$$

By (3.3),

$$
z^{T} K^{2} f\left(c^{T} x\right)=\left(K^{2} z-c^{T} x+c^{T} x\right)^{T} f\left(c^{T} x\right) \geq\left|f\left(c^{T} x\right)\right|\left(k\left|c^{T} x\right|-\left|K^{2} z-c^{T} x\right|\right),
$$

so (3.7) holds for $(x, z)$ belonging to the cone $C=\left\{(x, z):\left|K^{2} z-c^{T} x\right| \leq\right.$ $\left.k\left|c^{T} x\right|\right\}$.

Observe that $L=\left\{(x, z): x=-F^{-1} b w, z=w, w \in R^{m}\right\}$ is a subspace of $R^{m n}$ spanned by eigenvectors of $A$ corresponding to $\lambda=0$. Since $-c^{T} F^{-1} b=A_{n-1}^{-1}$, then $K^{2}+c^{T} F^{-1} b=0$, and direct calculation yields $(L \backslash\{0\}) \subset$ Int $C$, which proves Theorem 2 with the assumption (3.4).

In the case $(3.5)$, replace $(3.1)$ by

$$
x^{\prime}=F x+b z, \quad z^{\prime}=-f\left(c^{T} x\right)+p(t),
$$

where $x, b, c, F$ are as before.

Since (3.8) is obtained from (3.6) by taking $p_{1}(t)=0$ and replacing $f\left(c^{T} x\right)$ by $f_{2}(t, x, z)=f\left(c^{T} x\right)-p(t)$, it remains only to verify that $f_{2}$ satisfies (2.2).

By (3.5), there is a $k_{1} \in(0, k)$ such that $y^{T}(f(y)-p(t)) \geq\left(k-k_{1}\right)|y|$. $|f(y)|$, which implies that $-z^{T} K^{2}\left(f\left(c^{T} x\right)-p(t)\right) \leq 0$ for $(x, z)$ in the cone $\left\{(x, z):\left|K^{2} z-c^{T} x\right| \leq\left(\left(k-k_{1}\right) /(1+k)\right)\left|c^{T} x\right|\right\}$; this completes the proof of Theorem 2 .

In the scalar case, instead of (3.1) one can consider a slightly more 
general equation

$$
y^{(n)}+g_{1}\left(y^{\prime(n-2)}\right) y^{(n-1)}+\cdots+g_{n-1}(y) y^{\prime}+f\left(y ; y^{\prime}, \cdots, y^{(n-1)}\right)=p(t),
$$

where $g_{i}: R \rightarrow R, f: R \times R^{n-1} \rightarrow R, p: R \rightarrow R$ are continuous. An application of Theorem 2 gives

Theorem 3. Let $G_{i}(u)=\int_{0}^{u} g_{i}(s) d s$ satisfy

$$
\left|G_{i}(u)-a_{i} u\right| \leq \mu|u| \text { for } i=1, \cdots, n-1,|u| \geq u_{1} \text {. }
$$

Assume that $a_{n-1}>0$ and that the polynomial $\phi(\lambda)=\lambda^{n-1}+a_{1} \lambda^{n-2}+$ $\cdots+a_{n-1}$ has roots different from $(2 \pi i / \omega) p, p=1,2, \cdots$. Let

$$
0<y f(y, x)<\mu y^{2} \text { for }|y| \geq y_{1}, z \in R^{n-1} \text {. }
$$

Let $p$ be $\omega$-periodic and either

$$
\int_{0}^{\omega} p(s) d s=0
$$

or

$$
|f(y, z)| \geq f_{0}>\max \{|p(t)|: t \in[0, \omega]\} \text { for }|y| \geq y_{1}, z \in R^{n-1} \text {. }
$$

If $\mu$ is sufficiently small, then (3.9) has at least one $\omega$-periodic solution.

Proof. Let $x_{1}=y, x^{T}=\left(x_{1}, \ldots, x_{n-1}\right)$ and

$$
G(x)=\left[a_{n-1} x_{1}-G_{n-1}\left(x_{1}\right)\right]+\cdots+\left[a_{1} x_{n-1}-G_{1}\left(x_{n-1}\right)\right],
$$

and let $b, c, F$ be as in Theorem $2(m=1)$.

Then (3.9) may be replaced by

$$
x^{\prime}=F x+b z+F_{1}(t, x, z), \quad z^{\prime}=F_{2}(t, x, z),
$$

where

$$
\begin{aligned}
& F_{1}(t, x, z)=b \int_{0}^{t} p(s) d s+b G(x), \\
& F_{2}(t, x, z)=-f\left(c^{T} x ; F x+b z+F_{1}(t, x, z)\right)
\end{aligned}
$$

if $p$ satisfies (3.13), and

$$
F_{1}(t, x, z)=b G(x), \quad F_{2}(t, x, z)=-f\left(c^{T} x ; F x+b z+F_{1}(t, x, z)\right)+p(t)
$$

otherwise. It is easily seen that the proof repeats arguments used above, hence it is left to the reader.

Theorem 3 generalizes results of [5] and partially of [6]. 


\section{REFERENCES}

1. M. A. Krasnosel'skii, The operator of translation along the trajectories of differential equations, "Nauka", Moscow, 1966; English transl., Transl. Math. Monographs, vol. 19, Amer. Math. Soc., Providence, R.I., 1968. MR 34 \#3012; $36 \# 6688$.

2. V. A. Pliss, The existence of periodic solutions of certain nonlinear systems, Dokl. Akad. Nauk SSSR 137 (1961), 1060-1062 = Soviet Math. Dokl. 2 (1961), 416-418. MR 22 \#9665.

3. R. Reissig, Note on a certain non-autonomous differential equation, Atti Accad. Naz. Lincei. Rend. Cl. Sci. Fis. Mat. Natur. (8) 48 (1970), 484-486. MR 44 \#1879.

4. - Periodic solutions of a nonlinear $n$-th order vector differential equation, Ann. Mat. Pura Appl. (4) 87 (1970), 111-123. MR 44 \#542.

5. R. Reissig, Periodic solutions of a third order nonlinear differential equation, Ann. Mat. Pura. Appl。92 (1972), 193-198.

6. - An extension of Ezeilo's result, Ann. Mat. Pura. Appl. 92 (1972), 199-210. 1969.

7. J. T. Schwartz, Nonlinear functional analysis, Gordon and Breach, New York,

8. S. Sedziwy, Asymptotic properties of solutions of nonlinear differential equations of the higher order, Zeszyty Nauk. Uniw. Jagiello. Prace Mat. 11 (1966), 69-80. MR 44 \#547.

9. - Asymptotic properties of solutions of a certain n-th order vector differential equation, Atti Accad. Naz. Lincei Rend. Cl. Sci. Fis. Mat. Natur. (8) 47 (1969), 472-475. MR $42 \# 6366$.

10. Periodic solutions of differential equations in the cylindrical space, Ann. Polon. Math. 26 (1972), 335-339. MR $46 \# 7637$.

DE PARTMENT OF MA THEMATICS, UNIVERSITY OF UTAH, SALT LAKE CITY, UTAH 84112

Current address: Instytut Matematyczny, Uniwersytet Jagielloński, Kraków, Reymonta 4, Poland 